\title{
Resveratrol ameliorates experimental periodontitis in diabetic mice through negative regulation of TLR4 signaling
}

\author{
Lei ZHEN ${ }^{1}$, De-sheng FAN ${ }^{2, *}$, Yan ZHANG ${ }^{3, *}$, Xin-ming $\mathrm{CAO}^{1}$, Li-ming WANG ${ }^{1}$ \\ ${ }^{1}$ Department of Periodontology, Shanghai Stomatological Disease Center, Shanghai 200001, China; ${ }^{2}$ Department of Pathology, \\ Shanghai Tongji Hospital, Tongji University School of Medicine, Shanghai 200065, China; ${ }^{3}$ Laboratory of Oral Biomedical Science and \\ Translational Medicine, School of Stomatology, Tongji University, Shanghai 200072, China
}

Aim: To investigate the therapeutic effects of resveratrol (RSV) on periodontitis in diabetic mice and to explore the underlying mechanisms in vitro.

Methods: Experimental periodontitis was induced in $d b / d b$ mice by ligature application of porphyromonas gingivalis. The mice were treated with RSV $(20 \mathrm{mg} / \mathrm{kg}$, po) daily for 4 weeks. Alveolar bone loss, proinflammatory cytokines and TLR4 expression in the gingival tissue were measured. Cultured gingival epithelial cells (GECs) were used for in vitro studies. The transcriptional activity of TLR4 downstream signaling was analyzed using Western blotting.

Results: RSV administration significantly decreased the blood glucose levels, and ameliorated alveolar bone loss in $d b / d b$ mice with experimental periodontitis. RSV administration also suppressed the high levels of IL-1 $\beta$, IL-6, IL-8, TNF- $\alpha$, and TLR4 in gingival tissue of the mice. In the GECs incubated in high glucose medium, TLR4 expression was substantially upregulated, which was partly blocked in the presence of RSV. Lipopolysaccharides markedly increased the expression and secretion of IL-1 $\beta$, IL-6, IL-8, and TNF- $\alpha$ in the GECs cultured in high glucose medium, which was also partly blocked in the presence of RSV. Furthermore, RSV significantly suppressed the phosphorylation of TLR4 downstream factors NF-KB p65, p38MAPK, and STAT3.

Conclusion: RSV exerts protective effects against experimental periodontitis in $d b / d b$ mice via negative regulation of TLR4 signaling.

Keywords: resveratrol; diabetes; $d b / d b$ mice; periodontitis; porphyromonas gingivalis; gingival epithelial cell; proinflammatory cytokine; TLR4; inflammation

Acta Pharmacologica Sinica (2015) 36: 221-228; doi: 10.1038/aps.2014.131; published online 22 Dec 2014

\section{Introduction}

Diabetes is a chronic metabolic disease that is characterized by hyperglycemia that results in complications such as retinopathy, microangiopathy, and nephropathy ${ }^{[1]}$. Chronic periodontitis is defined as an inflammatory disease that is caused by oral pathogenic bacteria. Strong evidence indicates that poorly controlled diabetes increases susceptibility to periodontitis, also known as diabetic periodontitis ${ }^{[2-4]}$. Patients with diabetic periodontitis exhibit greater alveolar bone loss and a poorer prognosis after routine treatments compared with patients who do not have diabetes ${ }^{[5]}$. Furthermore, some studies have revealed that an altered host immune response results in excessive inflammation and increases the severity of

\footnotetext{
* To whom correspondence should be addressed.

E-mail bingliyisheng@126.com (De-sheng FAN);

zhangyankq@tongji.edu.cn (Yan ZHANG)

Received 2014-08-05 Accepted 2014-10-30
}

periodontal tissue destruction in diabetic periodontitis ${ }^{[6,7]}$.

Toll-like receptors (TLRs) are a family of pattern recognition receptors that play a major role in the recognition of pathogenassociated molecular patterns in innate immunity ${ }^{[8,9]}$. TLR4, as a cellular receptor for bacterial lipopolysaccharide (LPS), is by far the most extensively studied member of the TLR family. LPS activates TLR4-induced downstream signaling molecules, including nuclear factor- $\mathrm{KB}(\mathrm{NF}-\mathrm{kB}), \mathrm{p} 38$ mitogen activated protein kinase (MAPK), c-Jun N-terminal kinase (JNK), and signal transducer and activator of transcription (STAT), leading to the production of proinflammatory cytokines ${ }^{[10]}$.

Some evidence indicates that the TLR4 levels are elevated in diabetes and its complications ${ }^{[11-13]}$. The increased expression of TLR4 induced by hyperglycemia results in the generation and secretion of a series of proinflammatory cytokines in adipocytes, monocytes, macrophages, and glomerular endothelial cells, among others ${ }^{[14,15]}$. Correspondingly, related complications commonly develop ${ }^{[16,17]}$. Recently, a higher expression 
of TLR4 has been found in gingival tissue from patients with diabetic periodontitis compared with tissue from patients who have chronic periodontitis but do not have diabetes ${ }^{[18,19]}$. In addition, TLR4 expression is markedly increased in gingival epithelial cells incubated with high glucose ${ }^{[20]}$. These findings suggest that TLR4 may act as a molecular signaling to link diabetes and periodontitis.

Resveratrol (RSV) is a naturally occurring polyphenolic phytoalexin that is produced by polygonum cuspidatum, mulberry (Morus species), grapes, and red wine. It has diverse biological activities including anti-oxidation ${ }^{[21]}$, anti-cancer ${ }^{[22]}$, anti-inflammation ${ }^{[23]}$, cardioprotection ${ }^{[24]}$, and neuroprotection $^{[25]}$. Several reports indicate that RSV is capable of preventing and treating diabetes and its related complications ${ }^{[26,27]}$. However, the effect of RSV on diabetic periodontitis and the underlying mechanisms of action are not clear. In this study, we hypothesize that RSV may decrease local periodontal tissue inflammation and thereby alleviate alveolar bone loss by inhibiting TLR4 expression in gingival tissue during diabetic periodontitis. To confirm this hypothesis, we employed an experimental periodontitis model to detect alveolar bone loss, proinflammatory cytokines and TLR4 expression in the gingival tissue of diabetic mice with or without RSV treatment. The possible molecular mechanisms were also explored in vitro.

\section{Materials and methods}

\section{Animals and experimental design}

C57BLKS/J- $d b / d b$ male mice, a model for type 2 diabetes, were obtained from the National Resource Center of Model Mice (Nanjing, China). All animal experiments were performed according to the USA National Institute of Health Guide for the Care and Use of Laboratory Animals, and the protocols were approved by the Ethics Committee for Experimental Research, Medical College of Tongji, Tongji University. These $d b / d b$ mice (6 weeks old; weight 30-33 g) were kept in a room with $12 \mathrm{~h}$ light-dark cycles and fed a standard laboratory Altromin chow. At 8 weeks of age, $d b / d b$ mice were randomly divided into 3 groups ( $n=10 /$ group): untreated control group (DC), experimental periodontitis group (DP), experimental periodontitis with RSV group (DPR).

Porphyromonas gingivalis strain (ATCC 33277) was purchased from the American Type Culture Collection (ATCC, Manassas, USA) and grown in an anaerobic chamber with $85 \% \mathrm{~N}_{2}$, $10 \% \mathrm{H}_{2}$, and $5 \% \mathrm{CO}_{2}$ at $37^{\circ} \mathrm{C}$. To induce experimental periodontitis, cotton ligatures presoaked in a medium containing porphyromonas gingivalis $\left(10^{8} / \mathrm{mL}\right)$ were wrapped around the cervical position of the maxillary first molars and knotted distal-buccally in the DP and DPR groups of mice. Ligatures were changed every other day. At the same time, mice in the DPR group received a gavage of RSV (Adipogen Corp, USA) at dose of $20 \mathrm{mg} / \mathrm{kg}$ body weight every day. Mice in the DP group received a similar volume of placebo via gavage. Mice in the DC group received neither the periodontal ligature nor any placebo. The animal experiment lasted for 4 weeks after the initial ligature application. At the end of these experiments, the fasting blood glucose levels of all mice were mea- sured using a glucometer.

\section{Alveolar bone loss measurement}

After euthanasia, mandibular jaws were dissected from surrounding soft tissues, immersed overnight in 3\% hydrogen peroxide, and stained with $1 \%$ methylene blue for $10 \mathrm{~min}$. The bone loss level of the first molars in each mouse was calculated by measuring the distance from the cementoenamel junction to the alveolar crest at six sites: mesio-buccal, mid-buccal, disto-buccal, mesio-palatal, mid-palatal, and disto-palatal. The alveolar bone loss data represent the mean in millimeters of the six measured sites.

\section{Gingival epithelial cell culture}

Gingival tissues were collected from 8-week-old C57BL/6 male mice (Shanghai Experimental Animal Center, Shanghai, China). The cells were isolated and cultured as described previously $^{[20]}$. Briefly, gingival tissue was cut into small pieces and incubated with dispase and trypsinase for $4 \mathrm{~h}$ to produce a single cell suspension. The cells were collected and resuspended in K-SFM medium (Sciencell,CA,USA) supplemented with $100 \mathrm{IU} / \mathrm{mL}$ penicillin and $100 \mu \mathrm{g} / \mathrm{mL}$ streptomycin (Gibco, USA). The medium was changed every $2 \mathrm{~d}$. The cells were used at passage 3 . At the indicated time points, cells were treated with $25 \mathrm{mmol} / \mathrm{L}$ glucose in the high glucose group. GECs were cultured in $5.5 \mathrm{mmol} / \mathrm{L}$ glucose in the control group.

\section{Quantitative real-time PCR (qRT-PCR) analysis}

Total RNA was extracted from gingival tissue samples and GECs using Trizol (Invitrogen, USA) according to the manufacturer's protocol. Synthesis of first-strand cDNA was carried out using an RT-PCR first-strand cDNA synthesis kit (Invitrogen). Then, $1 \mu \mathrm{g}$ cDNA was used for real-time PCR in a BioRad iQ5 thermal cycler. The mRNA expression levels of the target genes were calculated via the comparative cycle threshold method using GAPDH as a control. The primer sequences used for real-time RT-PCR were as follows:

GAPDH: forward 5'-ACAGTCAGCCGCATCTTCTT-3', reverse 5'-GACAAGCTTCCCGTTCTCAG-3';

IL-1 $\beta$ : forward 5'-GCAACTGTTCCTGAACTCAACT-3', reverse 5'-ATCTTTTGGGGTCCGTCAACT-3';

IL-6: forward 5'-AGTTG CCTTCTTGGGACTGA-3', reverse 5'-CAGAATTGCCATTGCACAAC-3';

IL-8: forward 5'-GACATACTCCAAACCTTTCCACC-3', reverse 5'-AACTTCTCCACAACCCTCTGC-3';

TNF-a: forward 5'-GTGGAACTGGCAGAAGAGGC-3', reverse 5'-AGACAGAAGAGCGTGGTGGC-3';

TLR4: forward 5'-AATTCCTGCAGTGGGTCAAG-3', reverse 5'-AGGCGATACAATTCCACCTG-3'.

\section{Enzyme-linked immunosorbent assay (ELISA)}

At the third passage, GECs were incubated in $25 \mathrm{mmol} / \mathrm{L}$ glucose with or without RSV $(10 \mu \mathrm{mol} / \mathrm{L})$ for $24 \mathrm{~h}$ and were subsequently treated with LPS from $P$ gingivalis at $100 \mathrm{ng} / \mathrm{mL}$ with or without RSV $(10 \mu \mathrm{mol} / \mathrm{L})$ for $2 \mathrm{~h}$. The levels of IL-1 $\beta$, 
IL-6, IL-8, and TNF- $\alpha$ in the culture media were measured using ELISA kits (R\&D, USA) according to the manufacturer's instructions.

\section{Western blot analysis}

The whole protein extraction and Western blot analyses were performed as previously described ${ }^{[28]}$. Briefly, cells were lysed on ice for $30 \mathrm{~min}$ with RIPA lysis buffer including protease and phosphatase inhibitors (Sigma, St Louis, MO, USA). Proteins were then extracted by centrifuging for $10 \mathrm{~min}$ at $10000 \times \mathrm{g}$ at $4{ }^{\circ} \mathrm{C}$. Then, $10 \mu \mathrm{g}$ of each sample protein were resuspended and electrophoresed on $8 \%-10 \%$ SDS-polyacrylamide gels. Fractionated proteins were transferred onto polyvinylidene difluoride membranes (Invitrogen, San Diego, CA,USA). After the membranes were blocked with $5 \%$ milk for $1 \mathrm{~h}$, they were incubated with primary antibodies (Santa Cruz, Dallas, TX, USA) overnight at $4{ }^{\circ} \mathrm{C}$. The secondary antibodies (goat-anti-rabbit IgG conjugated with horseradish peroxidase, Santa Cruz, Dallas, TX, USA) were added to the membranes and incubated for $1 \mathrm{~h}$ at room temperature. Reactive protein bands were detected using Western Blotting Luminol Reagent (Invitrogen, San Diego, CA, USA) and exposed to X-ray film.

\section{Statistical analysis}

The SPSS 17.0 software was used to complete data processing. All data are represented as the mean $\pm S D$ of three independent experiments. Statistical significance was tested by Student's $t$-test and one-way ANOVA. The results were considered statistically significant when $P<0.05$.

\section{Results}

RSV ameliorates experimental periodontitis in $d b / d b$ mice

To examine the therapeutic effects of RSV on diabetic periodontitis, we established experimental periodontitis in $d b / d b$ mice with or without RSV treatment. First, we measured the fasting blood glucose levels of diabetic mice with or without RSV treatment. Although all mice had hyperglycemia, RSV-treated mice had lower blood glucose levels than did untreated mice with experimental periodontitis (DPR group vs DP group). The level of fasting blood glucose was not considerably different between mice with or without experimental periodontitis (DP group vs DC group) (Figure 1A). Conversely, in terms of alveolar bone loss, our results showed that there was a significant increase in alveolar bone loss in experimental periodontitis mice compared with control mice (DP group ws DC group). However, RSV treatment remarkably decreased alveolar bone loss associated with experimental periodontitis in $d b / d b$ mice (DPR group vs DP group) (Figure $1 \mathrm{~B})$. It is well known that proinflammatory cytokines play an important role in periodontal tissue breakdown. We next determined whether RSV affected the expression of proinflammatory cytokines in gingival tissue. Our results showed that RSV treatment markedly decreased the mRNA expression of the proinflammatory cytokines IL-1 $\beta$, IL-6, IL- 8 , and TNF- $\alpha$ in the gingival tissue of mice with experimental periodontitis (Figure 1C).

\section{RSV reduces TLR4 expression in vivo and in vitro}

qRT-RCR and Western blot were performed to detect TLR4 expression in the gingival tissue of experimental periodontitis mice treated with RSV or untreated. It was revealed that there were significant differences in the TLR4 mRNA and protein levels in the gingival tissue of diabetic periodontitis mice (DP group) and diabetic control mice (DC group). However, RSV treatment significantly reduced the expression of TLR4 in the gingival tissue of diabetic periodontitis mice (DPR group vs DP group) (Figure 2A).

In the in vitro experiment, GECs were exposed to 5.5 $\mathrm{mmol} / \mathrm{L}$ glucose (NG), $25 \mathrm{mmol} / \mathrm{L}$ glucose (HG) or 25 $\mathrm{mmol} / \mathrm{L}$ glucose with $10 \mu \mathrm{mol} / \mathrm{L} \mathrm{RSV}(\mathrm{HG}+\mathrm{RSV})$ for $24 \mathrm{~h}$. It can be observed that the TLR4 mRNA and protein expressions were higher in those cells incubated in $25 \mathrm{mmol} / \mathrm{L}$ glucose (HG) compared with those cultured in $5.5 \mathrm{mmol} / \mathrm{L}$ glucose (NG). However, RSV treatment (HG+RSV) significantly reduced the expression of TLR4 induced by high glucose (Figure $2 \mathrm{~B})$.

RSV downregulates LPS-TLR4-induced proinflammatory cytokines gene expression and protein secretion in GECs

To evaluate whether the effects of RSV on proinflammatory cytokines were mediated by TLR4 signaling, GECs were pretreated with $25 \mathrm{mmol} / \mathrm{L}$ glucose with or without RSV (10 $\mu \mathrm{mol} / \mathrm{L}$ ) for $24 \mathrm{~h}$ and subsequently treated with fresh media containing $P$ gingivalis LPS at $100 \mathrm{ng} / \mathrm{mL}$ with or without RSV $(10 \mu \mathrm{mol} / \mathrm{L})$ for $2 \mathrm{~h}$. The total RNA and culture supernatants were collected for real-time PCR and ELISA analysis, respectively. Our results indicated that RSV significantly suppressed the LPS-TLR4-mediated upregulation of IL-1 $\beta$, IL-6, IL-8, and TNF- $\alpha$ mRNA expression and protein secretion under high glucose conditions (Figure 3).

\section{RSV inhibits LPS-TLR4-induced activation of NF-KB p65, p38} MAPK, and STAT3

To understand the underlying molecular mechanisms of RSV downregulation of LPS-TLR4-induced proinflammatory cytokine expression, the activation of TLR4 downstream signaling molecules NF-kB p65, p38 MAPK, and STAT3 was determined by Western blot analysis. Cells were pre-treated with 25 mmol/L glucose with or without RSV $(10 \mu \mathrm{mol} / \mathrm{L})$ for $24 \mathrm{~h}$ and then treated with fresh media containing $P$ gingivalis LPS (100 ng/mL) with or without RSV $(10 \mu \mathrm{mol} / \mathrm{L})$ for 30 and 60 min. Our findings showed that RSV significantly inhibited the LPS-induced phosphorylation of NF-kB p65, p38 MAPK, and STAT3, which act as downstream molecules of the TLR4 signaling pathway (Figure 4).

\section{Discussion}

Diabetes is a chronic condition characterized by hyperglycemia, which exhibits an increased risk and severity to periodontal disease than that without diabetes ${ }^{[2.3]}$. The main explanation for the occurrence of aggravating periodontitis in diabetic patients is the disrupted immuno-inflammatory homeostasis during bacteria-host interactions. RSV is a natural polypheno- 
A

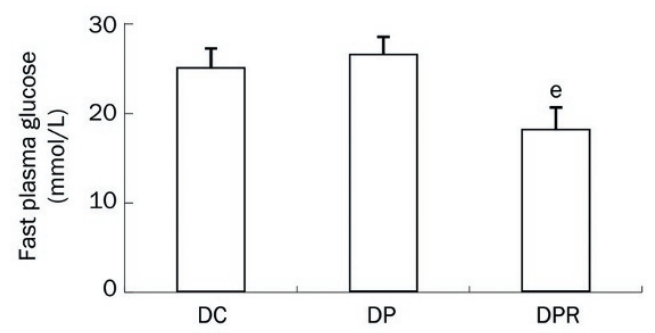

B
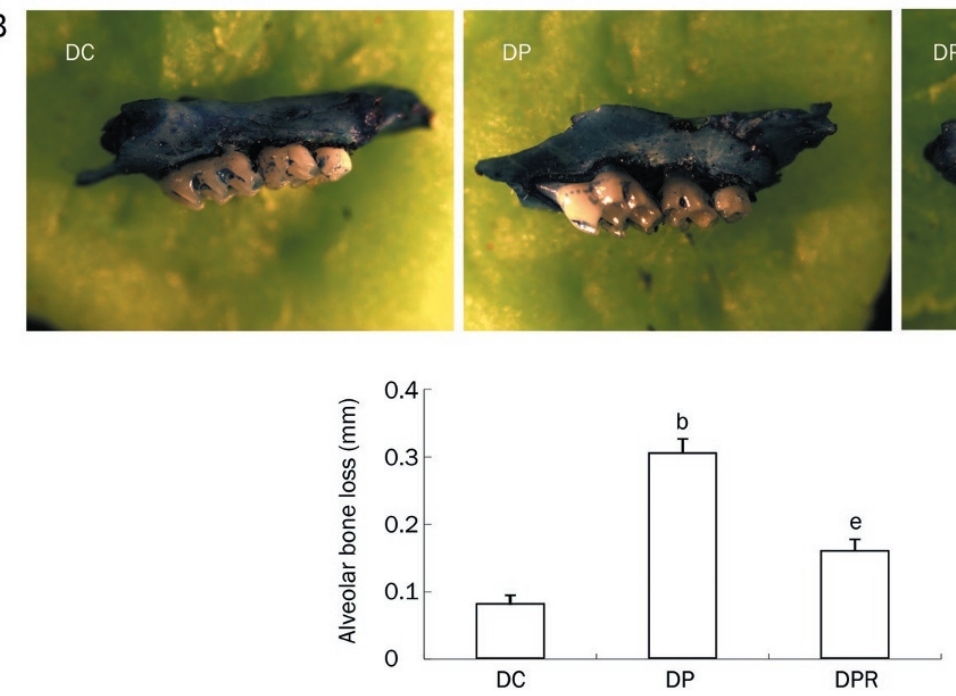

C

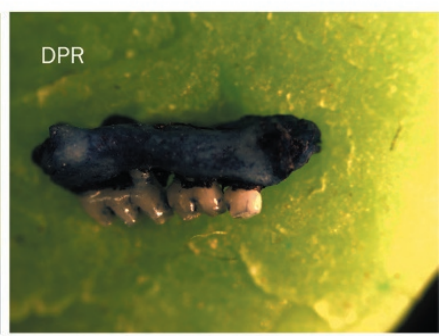

DPR 
A
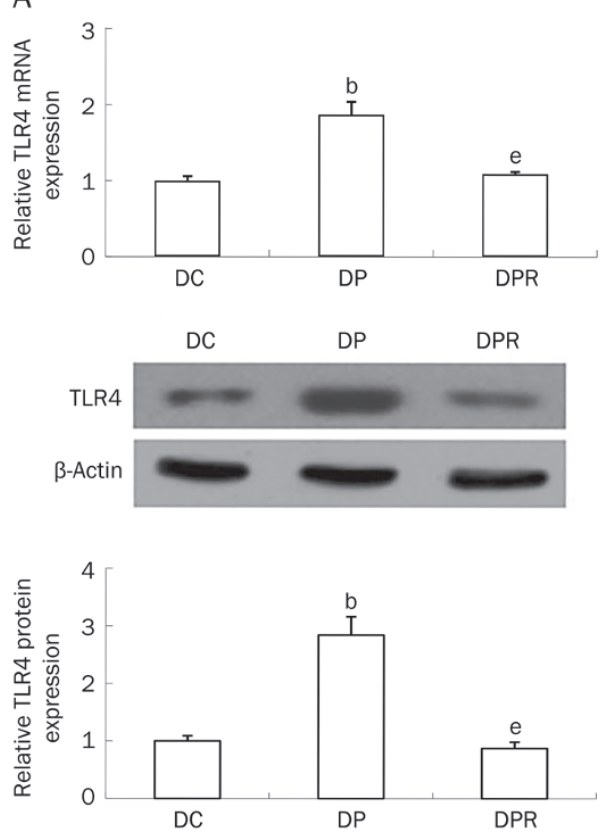

B
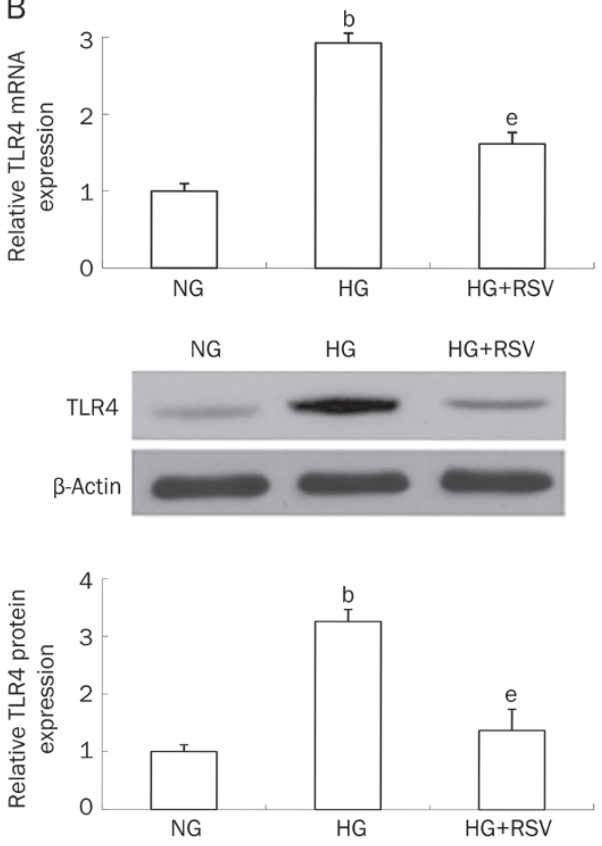

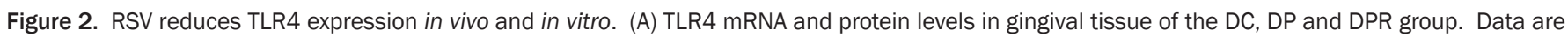

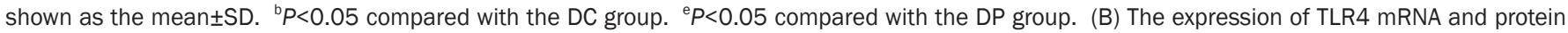

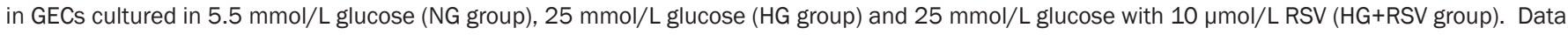
are shown as the mean \pm SD. $n=3$. ${ }^{b} P<0.05$ compared with the NG group. ${ }^{\mathrm{e}} P<0.05$ compared with the $\mathrm{HG}$ group.

this in vivo study suggested that RSV is potentially beneficial for treating diabetic periodontitis based on its dual roles in decreasing blood glucose levels and inhibiting proinflammatory cytokine production.

Toll-like receptors (TLRs) are pivotal innate immune receptors that induce inflammatory responses in diabetes. TLR2 and TLR4 expression and activation are increased in bone marrow-derived macrophages of non-obese diabetic (NOD) mice, correlating with increased NF-kB activation and increased pro-inflammatory cytokines ${ }^{[33]}$. A clinical study showed that TLR2/4 mediated inflammation in monocytes was significantly correlated to the HbA1c levels in diabetic patients ${ }^{[34]}$. Furthermore, TLR4 has been reported to link the inflammatory responses of diabetes and periodontitis. Increased expression of TLR 4 was observed in the gingival epithelial tissue during hyperglycemia ${ }^{[35]}$, and the TLR4 levels were upregulated in diabetic periodontitis subjects compared with periodontally healthy subjects with diabetes ${ }^{[19]}$. A recent study by Yang $X$ et al found that TLR4 expression increased in human gingival epithelial cells (HGECs) treated with high glucose in vitro ${ }^{[20]}$. Activation of the TLR4 signaling pathway in gingival epithelial cells led to the production of several proinflammatory cytokines, including IL-6, IL-8, and TNF-a, and triggered periodontal tissue destruction. Therefore, TLR4 is an attractive target of host modulation therapy for diabetic periodontitis. Recently, some evidence has demonstrated that RSV can restore TLR4-induced inflammatory responses in monocytes ${ }^{[36]}$. These results suggest an association between TLR4 and RSV regulatory functions in inflammation. In the current study, increased TLR4 expression in the gingival epithelium was found in diabetic periodontitis mice, whereas RSV markedly reduced TLR4 expression in $\mathrm{db} / \mathrm{db}$ mice with periodontitis. The subsequent study using cultured gingival epithelial cells under high glucose conditions also supported the finding that RSV reduced TLR4 expression and downregulated LPS-TLR4-induced proinflammatory cytokine production. This study is the first to demonstrate the inhibitory effect of RSV on TLR4 expression levels and TLR4-induced proinflammatory cytokine production in the gingival tissue of diabetic periodontitis mice and in gingival epithelial cells under high glucose conditions.

It has been reported that binding of LPS to the TLR4 receptor triggers activation of the NF- $\mathrm{B}$, MAPKs, and JAK-STAT signaling pathways ${ }^{[37]}$. To further explore the mechanisms behind the effects of RSV on diabetic periodontitis, this study focused on the TLR4 downstream signaling molecules NF- $\mathrm{kB}$ p65, p38 MAPK, and STAT3. NF-кB, known as a master transcription factor, regulates a number of proinflammatory cytokines and contributes to the development of inflammatory diseases. LPS-activated MAPK pathways, including the phosphorylation of p38 and JNK, lead to the production of several inflammatory mediators. Some studies have also demonstrated that the crosstalk between TLR4 and the JAK/STAT pathway contributes to the progression of diabetic periodontitis $^{[38]}$. In the present study, we observed that RSV had an inhibitory effect on LPS-activated NF-kB, p38 MAPK, and STAT3 pathways in gingival epithelial cells. Taken together, our results suggested that RSV suppressed inflammatory 
A
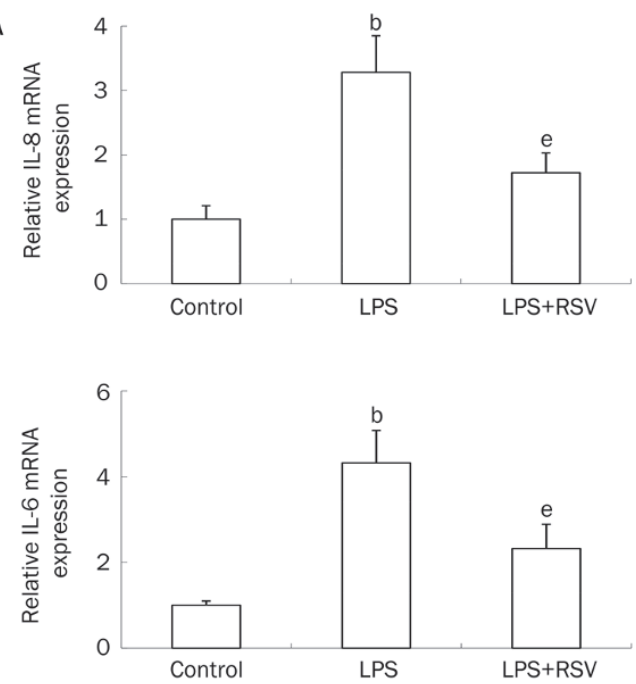

B
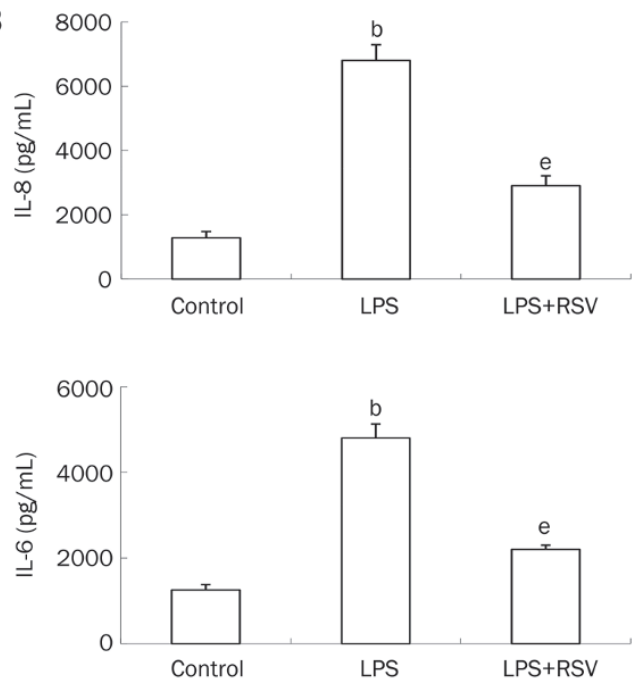
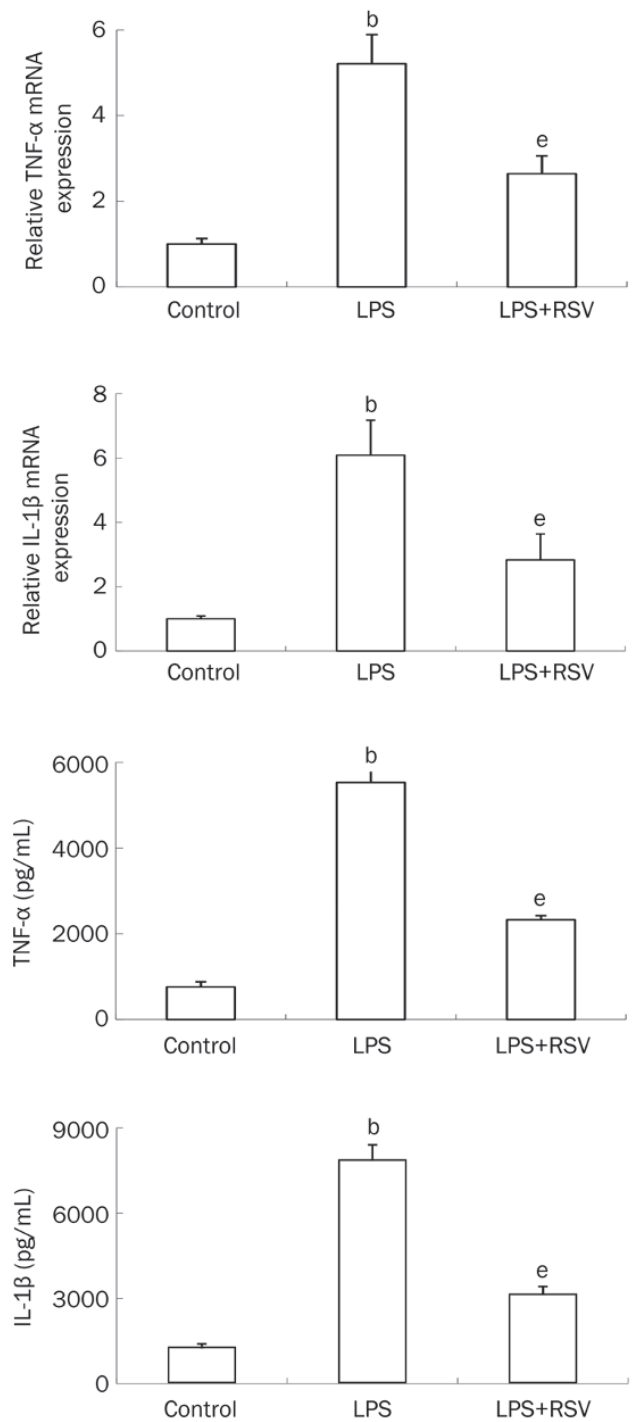

Figure 3. RSV downregulates LPS-TLR4-induced proinflammatory cytokine gene expression and protein secretion in GECs. GECs were pre-treated with $25 \mathrm{mmol} / \mathrm{L}$ glucose with or without RSV $(10 \mu \mathrm{mol} / \mathrm{L})$ for $24 \mathrm{~h}$ and subsequently treated with LPS at $100 \mathrm{ng} / \mathrm{mL}$ with or without RSV (10 $\mu \mathrm{mol} / \mathrm{L})$ for $2 \mathrm{~h}$. (A) IL-1 $\beta, I L-6, I L-8$, and TNF- $\alpha$ mRNA expression. (B) IL-1 $\beta, I L-6, I L-8$, and TNF- $\alpha$ protein secretion. Data are shown as the mean $\pm S D$. $n=3$. ${ }^{b} P<0.05$ compared with the control. ${ }^{\mathrm{e}} \mathrm{P}<0.05$ compared with the LPS group.

mediator expression via the negative regulation of TLR4 and downstream signaling molecules, including NF-KB, p38 MAPK, and STAT3.

In conclusion, the current study provides evidence for the inhibitory effect of RSV on hyperglycemia and alveolar bone loss in diabetic periodontitis. These results also demonstrate that RSV can attenuate the inflammatory response in diabetic periodontitis by downregulating TLR4 expression and TLR4 downstream signaling activation. All of the aforementioned findings suggest that RSV may potentially serve as a therapeutic agent against diabetic periodontitis.

\section{Acknowledgements}

This study is supported by the Shanghai Natural Science Foundation (№ 13ZR1436300), the Young Scholars Project of the Shanghai Health Bureau (№ 20124y034), the Shanghai
Yong Doctor Program (№ 2012-04-03), and the Natural Science Foundation of the Shanghai Stomatological Disease Center (No SSDC-FZ-2012-01, SSDC-2012-05).

\section{Author contribution}

Lei ZHEN and De-sheng FAN performed most of the experiments, analyzed the data and drafted the manuscript; Xinming CAO and Li-ming WANG assisted in the experiments; Lei ZHEN and Yan ZHANG designed the study and reviewed the manuscript.

\section{References}

1 Imam K. Management and treatment of diabetes mellitus. Adv Exp Med Biol 2012; 771: 356-80.

2 Preshaw PM. Periodontal disease and diabetes. J Dent 2009; 37: S575-7. 

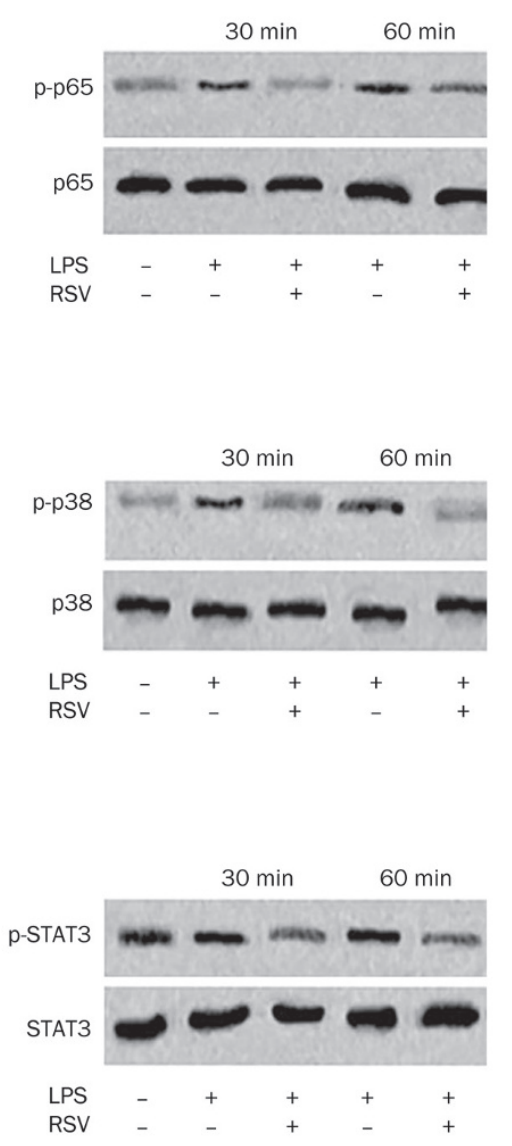
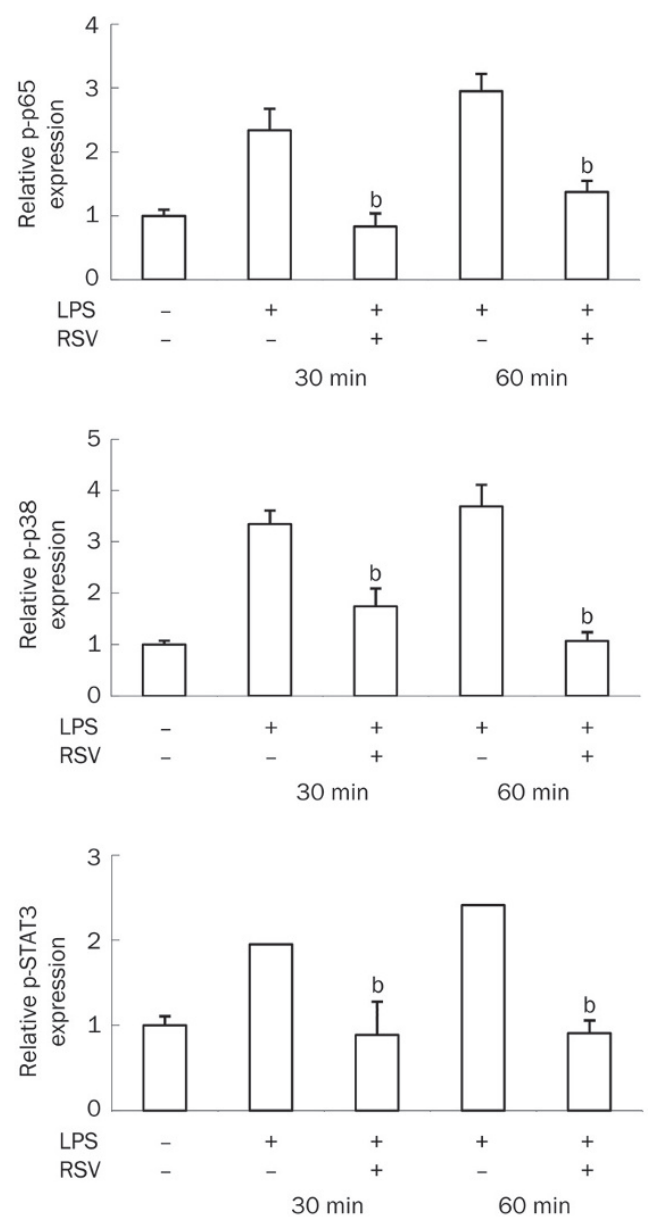

Figure 4. RSV inhibits activation of NF-KB p65, p38 MAPK, and STAT3. GECs were pre-treated with $25 \mathrm{mmol} / \mathrm{L}$ glucose with or without RSV (10 $\mu$ mol/L) for $24 \mathrm{~h}$ and then treated with LPS $(100 \mathrm{ng} / \mathrm{mL})$ with or without RSV $(10 \mu \mathrm{mol} / \mathrm{L})$ for 30 and $60 \mathrm{~min}$. The levels of phosphorylated and total p65, p38, and STAT3 were assessed by Western blot. Data are shown as the mean \pm SD. $n=3$. ${ }^{b} P<0.05$ compared with the LPS group.

3 Preshaw PM, Alba AL, Herrera D, Jepsen S, Konstantinidis A, Makrilakis $\mathrm{K}$, et al. Periodontitis and diabetes: a two-way relationship. Diabetologia 2012; 55: 21-31.

4 Almeida Abdo J, Cirano FR, Casati MZ, Ribeiro FV, Giampaoli V, Viana Casarin RC, et al. Influence of dyslipidemia and diabetes mellitus on chronic periodontal disease. J Periodontol 2013; 84: 1401-8.

$5 \mathrm{Kim} \mathrm{JH}$, Lee DE, Gunawardhana KS, Choi SH, Woo GH, Cha JH, et al. Effect of the interaction between periodontitis and type 1 diabetes mellitus on alveolar bone, mandibular condyle and tibia. Acta Odontol Scand 2014; 72: 265-73.

6 Sun WL, Chen LL, Zhang SZ, Wu YM, Ren YZ, Qin GM. Inflammatory cytokines, adiponectin, insulin resistance and metabolic control after periodontal intervention in patients with type 2 diabetes and chronic periodontitis. Intern Med 2011; 50: 1569-74.

7 Duarte PM, Bezerra JP, Miranda TS, Feres M, Chambrone L, Shaddox LM. Local levels of inflammatory mediators in uncontrolled type 2 diabetic subjects with chronic periodontitis.J Clin Periodontol 2014; 41: 11-8.

8 Trinchieri G, Sher A. Cooperation of toll-like receptor signals in innate immune defence. Nat Rev Immunol 2007; 7: 179-90.

9 Nogueira-Machado JA, Volpe CM, Veloso CA, Chaves MM. HMGB1, TLR, and RAGE: a functional tripod that leads to diabetic inflammation. Expert Opin Ther Targets 2011; 15: 1023-35.
10 Luo W, Wang CY, Jin L. Baicalin downregulates Porphyromonas gingivalis lipopolysaccharide-upregulated IL-6 and IL-8 expression in human oral keratinocytes by negative regulation of TLR signaling. PLoS One 2012; 7: e51008.

11 Dasu MR, Devaraj S, Park S, Jialal I. Increased toll-like receptor (TLR) activation and TLR ligands in recently diagnosed type 2 diabetic subjects. Diabetes Care 2010; 33: 861-8.

12 Devaraj S, Jialal I, Yun JM, Bremer A. Demonstration of increased toll-like receptor 2 and toll-like receptor 4 expression in monocytes of type 1 diabetes mellitus patients with microvascular complications. Metabolism 2011; 60: 256-9.

13 Dasu MR, Martin SJ. Toll-like receptor expression and signaling in human diabetic wounds. World J Diabetes 2014; 5: 219-23.

14 Takata S, Sawa Y, Uchiyama T, Ishikawa H. Expression of toll-like receptor 4 in glomerular endothelial cells under diabetic conditions. Acta Histochem Cytochem 2013; 46: 35-42.

15 Mudaliar H, Pollock C, Komala MG, Chadban S, Wu H, Panchapakesan $\mathrm{U}$. The role of Toll-like receptor proteins (TLR) 2 and 4 in mediating inflammation in proximal tubules. Am J Physiol Renal Physiol 2013; 305: F143-54.

16 Ma J, Chadban SJ, Zhao CY, Chen X, Kwan T, Panchapakesan U, et al. TLR4 activation promotes podocyte injury and interstitial fibrosis in diabetic nephropathy. PLoS One 2014; 9: e97985. 
17 Kaur H, Chien A, Jialal I. Hyperglycemia induces Toll like receptor 4 expression and activity in mouse mesangial cells: relevance to diabetic nephropathy. Am J Physiol Renal Physiol 2012; 303: F114550 .

18 Rojo-Botello NR, García-Hernández AL, Moreno-Fierros L. Expression of toll-like receptors 2, 4, and 9 is increased in gingival tissue from patients with type 2 diabetes and chronic periodontitis. J Periodontal Res 2012; 47: 62-73.

19 Promsudthi A1, Poomsawat S, Limsricharoen W. The role of toll-like receptor 2 and 4 in gingival tissues of chronic periodontitis subjects with type 2 diabetes. J Periodontal Res 2014; 49: 346-54.

20 Yang X, Zhang J, Ni J, Ouyang B, Wang D, Luo S, et al. Toll-like receptor 4 mediated hyper-responsiveness of gingival epithelial cells to LPS in high-glucose environment. J Periodontol 2014; 85: 1620-8.

21 Li W, Wang YP, Gao L, Zhang PP, Zhou Q, Xu QF, et al. Resveratrol protects rabbit ventricular myocytes against oxidative stress-induced arrhythmogenic activity and $\mathrm{Ca}^{2+}$ overload. Acta Pharmacol Sin 2013; 34: 1164-73.

22 Sun CY, Hu Y, Guo T, Wang HF, Zhang XP, He WJ, et al. Resveratrol as a novel agent for treatment of multiple myeloma with matrix metalloproteinase inhibitory activity. Acta Pharmacol Sin 2006; 27: $1447-$ 52.

23 Wang SJ, Bo QY, Zhao XH, Yang X, Chi ZF, Liu XW. Resveratrol pretreatment reduces early inflammatory responses induced by status epilepticus via mTOR signaling. Brain Res 2013; 1492: 122-9.

24 Magyar K, Halmosi R, Palfi A, Feher G, Czopf L, Fulop A, et al. Cardioprotection by resveratrol: a human clinical trial in patients with stable coronary artery disease. Clin Hemorheol Microcirc 2012; 50: 179-87.

25 Lin TK, Chen SD, Chuang YC, Lin HY, Huang CR, Chuang JH, et al. Resveratrol partially prevents rotenone-induced neurotoxicity in dopaminergic SH-SY5Y cells through induction of heme oxygenase-1 dependent autophagy. Int J Mol Sci 2014; 15: 1625-46.

26 Zeytin K, Ciloğlu NS, Ateş F, Vardar Aker F, Ercan F. The effects of resveratrol on tendon healing of diabetic rats. Acta Orthop Traumatol Turc 2014; 48: 355-62.

27 Guo R, Liu B, Wang K, Zhou S, Li W, Xu Y. Resveratrol ameliorates diabetic vascular inflammation and macrophage infiltration in $d b / d b$ mice by inhibiting the NF-KB pathway. Diab Vasc Dis Res 2014; 11 : 92-102.

28 Guo D, Li JR, Wang Y, Lei LS, Yu CL, Chen NN. Cyclovirobuxinum D suppresses lipopolysaccharide-induced inflammatory responses in murine macrophages in vitro by blocking JAK-STAT signaling pathway. Acta Pharmacol Sin 2014; 35: 770-8.

29 Su HC, Hung LM, Chen JK. Resveratrol, a red wine antioxidant, possesses an insulin-like effect in streptozotocin-induced diabetic rats. Am J Physiol Endocrinol Metab 2006; 290: E1339-46.

30 Zheng X, Zhu S, Chang S, Cao Y, Dong J, Li J, et al. Protective effects of chronic resveratrol treatment on vascular inflammatory injury in streptozotocin-induced type 2 diabetic rats: role of NF-kappa B signaling. Eur J Pharmacol 2013; 720: 147-57.

31 Mahmoud F, Al-Ozairi E. Inflammatory cytokines and the risk of cardiovascular complications in type 2 diabetes. Dis Markers 2013; 35: 235-41.

32 Maedler K, Sergeev P, Ris F, Oberholzer J, Joller-Jemelka HI, Spinas $\mathrm{GA}$, et al. Glucose-induced beta cell production of IL-1beta contributes to glucotoxicity in human pancreatic islets. J Clin Invest 2002; 110: 851-60.

33 Mohammad MK, Morran M, Slotterbeck B, Leaman DW, Sun Y, Grafenstein $\mathrm{H}$, et al. Dysregulated Toll-like receptor expression and signaling in bone marrow-derived macrophages at the onset of diabetes in the non-obese diabetic mouse. Int Immunol 2006; 18: 1101-13.

34 Ahmad R, Al-Mass A, Atizado V, Al-Hubail A, Al-Ghimlas F, Al-Arouj M, et al. Elevated expression of the toll like receptors 2 and 4 in obese individuals: its significance for obesity-induced inflammation. J Inflamm (Lond) 2012; 9: 48.

35 Becerik S, Ozsan N, Gürkan A, Oztürk VÖ, Atilla G, Emingil G. Toll like receptor 4 and membrane-bound CD14 expressions in gingivitis, periodontitis and CsA-induced gingival overgrowth. Arch Oral Biol 2011; 56: 456-65.

36 Jakus PB, Kalman N, Antus C, Radnai B, Tucsek Z, Gallyas F Jr, et al. TRAF6 is functional in inhibition of TLR4-mediated NF-KB activation by resveratrol. J Nutr Biochem 2013; 24: 819-23.

37 Zhang Z, Chen N, Liu JB, Wu JB, Zhang J, Zhang Y, et al. Protective effect of resveratrol against acute lung injury induced by lipopolysaccharide via inhibiting the myd88-dependent toll-like receptor 4 signaling pathway. Mol Med Rep 2014; 10: 101-6.

38 Wang Q, Li H, Xie H, Fu M, Guo B, Ding Y, et al. 25-Hydroxyvitamin D3 attenuates experimental periodontitis through downregulation of TLR4 and JAK1/STAT3 signaling in diabetic mice. J Steroid Biochem Mol Biol 2113; 135: 43-50. 